\title{
High Energy Break-Up of Few-Nucleon Systems
}

\author{
Misak Sargsian \\ Department of Physics, Florida International University, Miami, FL 33199
}

November 1, 2018

\begin{abstract}
Abstract We discus recent developments in theory of high energy two-body breakup reactions of few-nucleon systems. The characteristics of these reactions are such that the hard two-body quasielastic subprocess can be clearly separated from the accompanying soft subprocesses. We discuss in details the hard rescattering model (HRM) in which hard photodisintegration develops in two stages. At first, photon knocks-out an energetic quark which rescatters subsequently with a quark of the other nucleon. The latter provides a mechanism of sharing the initial high momentum of the photon by the outgoing two nucleons. Within HRM we discuss hard break-up reactions involving ${ }^{2} \mathrm{D}$ and ${ }^{3} \mathrm{He}$ targets. Another development of HRM is the prediction of new helicity selection mechanism for hard two-body reactions, which was apparently confirmed in the recent JLab experiment.
\end{abstract}

\section{Introduction to Nuclear QCD}

There is class of high-energy and momentum transfer (semi) exclusive nuclear reactions which suits very well for studies of Nuclear QCD. There are several common features in theoretical approaches for studying these reactions: (a) one can clearly identify a hard subprocess; (b) which can be factorized from the soft nuclear part of the reaction; (c) the subprocess is hard enough for pQCD to be applicable; (d) soft part of the reaction could be expressed through the measurable/extractable quantities such as partonic distribution functions, hadron-hadron scattering amplitudes, form-factors and calculable nuclear wave functions. If all above requirements are achieved then such theoretical approaches may yield (sometimes) parameter free predictions (see e.g. [1]) and practically always will allow us to study the aspects of strong interaction dynamics which otherwise can not be investigated without using nuclear targets.

Several of such reactions which satisfy above criteria are, (i) semiinclusive deep-inelastic nuclear reactions aimed at studies of nuclear modifications of PDFs (EMC effects)(see e.g. [2]); (ii) high momentum transfer elastic scattering off few-nucleon systems that can be used to study the onset of quark degrees of freedom in strongly correlated few nucleon systems [3], (iii) electrodisintegration of few-nucleon systems in the kinematics dominated by final state interaction that is used to probe the dominance of point like configurations in the hadronic wave function at large $Q^{2}$ (color transparency/coherence phenomena) [4, 5, 6, 7]; 


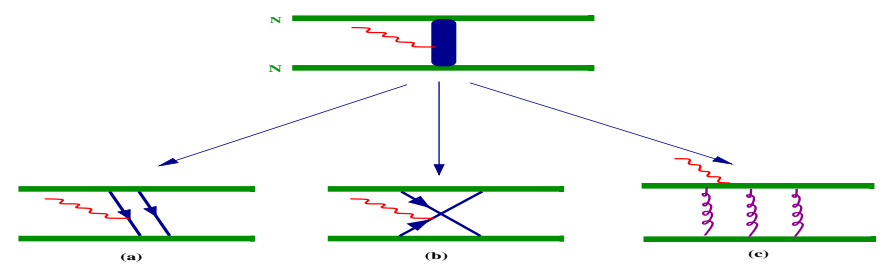

Figure 1: Possible QCD dynamics of NN interaction.

(iv) DIS nuclear scattering at $x_{B j}>1$ as a framework for investigation of the mechanism of generation of super-fast quarks[ㅇ, 2] (v) as well as, high-energy break-up of two nucleons in nuclei which can be used to probe the dynamics of strong interaction in two-nucleon system at intermediate to short distances [1, 3, 9, 10].

\section{High energy break-up of two nucleons in nuclei}

In this presentation we focus on the last class (v) of the reactions, in which high energy photon produces two energetic nucleons which equally share the initial energy of the photon. These reaction kinematically corresponds to the break-up of $2 \mathrm{~N}$ system at $90^{\circ}$ angle in the $\gamma-2 N$ center of mass reference frame.

Due to completely symmetric configuration, photon predominantly probes the structure and the dynamics of the exchanged particle in the NN system (Fig.1). High energy of photon in this case provides necessary resolution to probe the QCD content of NN interaction whether it proceeds through the $q \bar{q}$ exchange (Fig.1b), quark interchange (Fig.1c) or gluon exchange (Fig.1d).

Effectiveness of these processes in probing QCD aspects of nuclear interaction can be seen (for $\gamma d \rightarrow p n$ reactions) from the following kinematical considerations [3, 9, 10]) in which:

$$
s=\left(k_{\gamma}+p_{d}\right)^{2}=2 M_{d} E_{\gamma}+M_{d}^{2} ; \quad t=\left(k_{\gamma}-p_{N}\right)^{2}=\left(\cos \left(\theta_{c m}\right)-1\right) \frac{s-M_{d}^{2}}{2} .
$$

Simple estimate shows that already at $E_{\gamma}>2 \mathrm{GeV}$ the invariant momentum transfer $-t \mid 90^{\circ}>4 \mathrm{GeV}^{2}$ and invariant mass of the $N N$ system $M_{N N}=\sqrt{s}>2 \mathrm{GeV}$. These are conditions for which one expects an onset of quark degrees of freedom in the dynamics of strong interaction 11$]$.

One of the first theoretical predictions for high energy and large CM angle $\gamma d \rightarrow p n$ reactions was the prediction of the $s$ dependence of the differential cross section based on the quark counting rule[3], which yields: $\frac{d \sigma}{d t} \sim s^{-11}$. This prediction was experimentally confirmed already starting at $E_{\gamma}=1 \mathrm{GeV}$ for several set of experiments at SLAC[12, 13] and Jefferson Lab[14, 15, 16, 17].

The quark counting predictions are based on the hypothesis that the Fock states with minimal number of partonic constituents dominate in two-body large angle hard collisions 18 . Although successful in describing energy dependences of the number of hard processes, this hypothesis does not allow to make calculation of the absolute values of cross sections. Especially for reactions involving baryons, calculations within perturbative QCD underestimate 


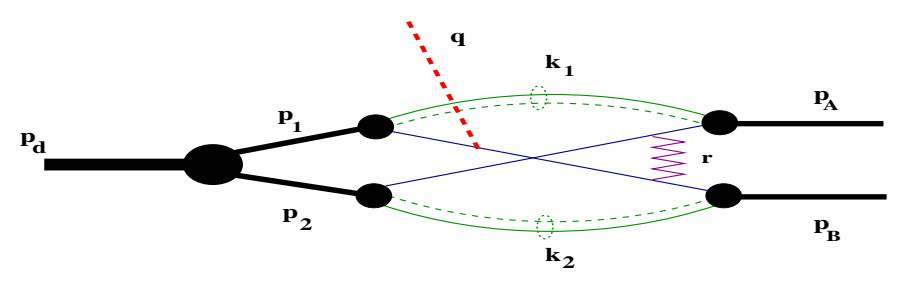

Figure 2: Typical diagram for hard rescattering mechanism.

the measured cross sections by orders of magnitude (see e.g.[19]). This may be an indication that in the accessible range of energies bulk of the interaction is in the domain of nonperturbative QCD [19, 20]. However, the main problem is that even if we fully realize the importance of nonperturbative interactions the theoretical methods of calculations in the nonperturbative domain are very restricted.

\section{Hard Rescattering Mechanism of Two-Body Break- up Reactions}

The underlying assumption in hard rescattering model (HRM) [1 is that high energy photodisintegration of two-nucleon system proceeds through the two stages in which an absorption of photon by a quark of one nucleon is followed by a high-momentum transfer (hard) rescattering with a quark from the second nucleon. The latter rescattering produces a final two nucleon state with large relative momenta. A typical diagram representing such a scenario is presented in Fig.2.

Analyzing the type of diagrams as in Fig.2 allows us to do the following observations:

- the dominant contribution comes from the soft vertices of $d \rightarrow N N$ transition, while quark rescattering proceeds trough the hard gluon exchange,

- the $d \rightarrow N N$ transition can be evaluated through the conventional deuteron wave functions,

- the structure of hard quark interchange interaction in the rescattering part of the reaction is similar to that of hard $\mathrm{NN}$ scattering,

- as a result the sum of the multitude of diagrams with incalculable nonperturbative part of the interaction can be expressed through the experimentally measured amplitude of hard $N N$ scattering.

Based on these observations, calculation of the $\gamma+d \rightarrow p n$ amplitude yields [1, 21]

$$
\begin{gathered}
\left\langle p_{\lambda_{A}}, n_{\lambda_{B}}|A| \lambda_{\gamma}, \lambda_{D}\right\rangle=\sum_{\lambda_{2}} \frac{f\left(\theta_{c m}\right)}{3 \sqrt{2 s^{\prime}}} \int \Psi^{\lambda_{D}, \lambda_{\gamma}, \lambda_{2}}\left(\alpha_{c}, p_{\perp}\right) \frac{d^{2} p_{\perp}}{(2 \pi)^{2}} \times \\
\left(\left\langle p_{\lambda_{A}}, n_{\lambda_{B}}\left|A_{p n}\left(s, t_{n}\right)\right| p_{\lambda_{\gamma}}, n_{\lambda_{2}}\right\rangle-\left\langle p_{\lambda_{A}}, n_{\lambda_{B}}\left|A_{p n}\left(s, u_{n}\right)\right| n_{\lambda_{\gamma}} p_{\lambda_{2}}\right\rangle\right),
\end{gathered}
$$



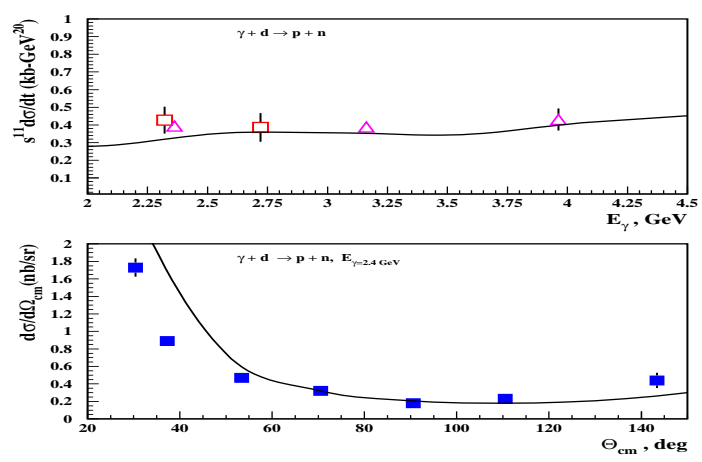

Figure 3: Energy dependence of the scaled cross section at $90^{\circ} \mathrm{CM}$ scattering (top) and angular dependence of the cross section at $E_{\gamma}=2.4 \mathrm{GeV}$ (bottom).

where $A_{p n}$ is high momentum transfer elastic $p n$ scattering amplitude, $\left|N_{\lambda}\right\rangle(N=p, n)$ represents the helicity wave function of nucleon and $\lambda_{\gamma}$ is the helicity of incoming photon. Based on Eq.(2) one obtains the following expression for the differential cross section of $\gamma+d \rightarrow p n$ reaction:

$$
\frac{d \sigma^{\gamma d \rightarrow p n}}{d t}=\frac{8 \alpha}{9} \pi^{4} \frac{1}{s^{\prime}} C\left(\frac{\tilde{t}}{s}\right) \frac{d \sigma^{p n \rightarrow p n}}{d t}\left|\int \Psi_{d}^{N R}\left(p_{z}=0, p_{t}\right) \sqrt{m_{n}} \frac{d^{2} p_{t}}{(2 \pi)^{2}}\right|^{2},
$$

where $s^{\prime}=s-4 m_{N}^{2}$ and $\tilde{t}=\left(p_{n}-m_{n}\right)^{2}$. The interesting properly of the function $C$ is that $C\left(\theta_{c m}=90^{\circ}\right) \approx 1$. Therefore for $90^{\circ} \mathrm{CM}$ scattering HRM prediction is parameter free. Since $p n$ cross section in high momentum transfer behaves like $s^{-10}$, Eq.(3) predicts same $s^{-11}$ dependence as quark counting rule without requiring an onset of pQCD regime. Also, due to angular dependence of the $p n$ amplitude, HRM predicts an angular distribution being not symmetric around $90^{\circ} \mathrm{CM}$. These predictions agree reasonably well with the experimental data[14, 16] (see e.g. Fig.3).

\section{Hard break-up of two protons from ${ }^{3} \mathrm{He}$}

With all its success and accuracies yet to be improved HRM is only one of the approaches in describing hard photodisintegration reactions. Other models such as reduced nuclear amplitude (RNA) formalism[24] and quark-gluon string (QGS) model[25] describe many features of hard photodisintegration reaction, with QGS being rather successful in describing lower energy data. However RNA and QGS require an absolute normalization.

Recently it was suggested[22, 23] that the break-up of pp pair from ${ }^{3} \mathrm{He}$ will further advance our understanding of the dynamics of hard photodisintegration of two-nucleon systems and allow further discrimination between above mentioned models.

Within HRM the typical diagram describing two-proton break-up is shown in Fig.4. HRM calculation similar to that of deuteron break-up reaction yields:

$$
\frac{d \sigma}{d t d^{3} p_{n}}=\left(\frac{14}{15}\right)^{2} \frac{16 \pi^{4} \alpha}{S-M_{3}^{2} H e}\left(\frac{2 c^{2}}{1+2 c^{2}}\right) \frac{d \sigma^{p p}}{d t}\left(s_{p p}, t_{n}\right) \frac{S_{34}}{E_{n}},
$$




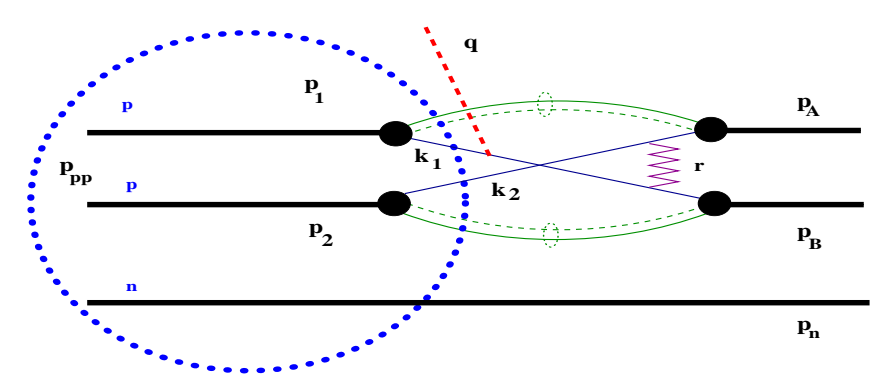

Figure 4: Typical diagram for hard break-up of pp pair from ${ }^{3} \mathrm{He}$.

where $S_{34}=\sum_{\lambda_{1}=-\lambda_{2}, \lambda_{3}=-\frac{1}{2}}^{\frac{1}{2}}\left|\int \psi_{3}^{\frac{1}{2}}{ }_{H e}\left(\lambda_{1}, \lambda_{2}, \lambda_{3}\right) m \frac{d^{2} p_{2 \perp}}{(2 \pi)^{2}}\right|^{2}$ and $c=\frac{\left|\phi_{3,4}\right|}{\left|\phi_{1}\right|}$ with $\phi_{i}$ being $p p$ helicity amplitudes. Since the cross section of $p p$ scattering enters in Eq.(4) one of the interesting predictions of HRM is the possibility of observation of energy oscillations at $90^{0} \mathrm{CM}$ scattering similar to one observed in elastic $p p$ scattering. Another interesting feature of two-proton break-up reactions is the fact that at lower energies this reaction is three-step [26] rather than two-step process. HRM in fact predicts that with an increase of energy due to the onset of quark-interchange (rather than meson-exchange) mechanism the two-body processes will dominate the cross section. The pioneering experiment of high energy $p p$ break-up reaction [27] was recently performed at Jefferson Lab which may shed new light on many issues of hard rescattering processes.

\section{Helicity Selection Mechanism}

In addition to the cross section measurements, polarization observables may provide a new insight into the dynamics of hard photodisintegration. Original motivation for polarization measurements in high energy photodisintegration reaction was the expectation that the onset of the pQCD regime in the reaction dynamics will be accompanied by an observation of the helicity conservation in polarized reaction. Both energy and angular distributions of several polarization observables have been measured at JLab[28, 29]. Although the energy range covered was rather restricted, it provided an interesting insight into the structure of HRM.

One of the unique features of HRM is that the struck quark carries the helicity of incoming photon. As a result one of the final nucleons will carry the bulk of the polarization of incident photon (see e.g. Eq.(2)). Thus in HRM photon plays as a helicity selector for the final nucleons. This yields a prediction for large asymmetry[27] $\left(C_{z^{\prime}}\right)$ for the longitudinal polarization of outgoing nucleons. In Ref.[21] we predicted a sizable asymmetry for $C_{z^{\prime}}$ even though the existing data[28] (with rather large errors) at that time were indicating on vanishing values of $C_{z^{\prime}}$. However, recent data[29], appears to confirm HRM prediction for large values of $C_{z^{\prime}}$ (see Fig.5). It will be interesting also to check the other HRM prediction that $C_{z^{\prime}}$ will continue to approach to unity with an increase of photon energy at $90^{0} \mathrm{CM}$ scattering.

It is very interesting that above described helicity selection mechanism of HRM predicts (an opposite) vanishing value of $C_{z^{\prime}}$ for two-body break up of proton pair from ${ }^{3} \mathrm{He}$. This follows from the fact that the dominant part of the amplitude which represents two final 


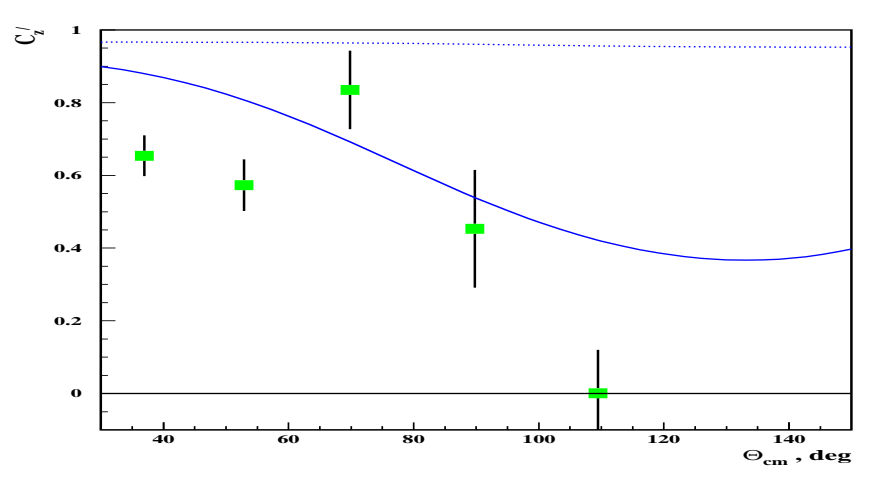

Figure 5: Angular dependence of $C_{z^{\prime}}$ for $E_{\gamma}=1.9 \mathrm{GeV}$.

state nucleons polarized in same direction are proportional to the nuclear ground state wave function with two initial nucleons having same helicities. Due to Pauli principle this part of the amplitude is strongly suppressed for the proton-pair in ${ }^{3} \mathrm{He}$ target. No such suppression exists for $p n$ break up reactions.

\section{Summary and Outlook}

There is an accumulating evidence that hard rescattering mechanism explains the underlying dynamics of high energy and large $\mathrm{CM}$ angle break-up of a nucleon pair from ${ }^{2} \mathrm{D}$ and ${ }^{3} \mathrm{He}$ targets. One of the important features of HRM is that its prediction of unpolarized cross section at $90^{\circ}$ center of mass photodisintegration of deuteron is parameter free and no further adjustments are required.

HRM predicts that energy dependence of two-proton break-up reaction should resemble that of hard elastic pp cross section.

Another feature of HRM, observed recently, is the prediction of large longitudinal asymmetries due to helicity selection mechanism characteristic to hard rescattering model.

If HRM will prove to be a true mechanism of hard photodisintegration reaction involving two nucleons, it will advance also our understanding of the dynamics of NN interaction at short distances.

A new venue for advancing our understanding of the dynamics of hard break-up reactions could be an extension of these studies to the kinematics in which two excited baryonic states (like $\Delta$-isobars) are produced at large center of mass angles of $\gamma-N N$ system.

\section{References}

[1] L.L. Frankfurt, G.A. Miller, M. M. Sargsian and M. I. Strikman, Phys. Rev. Lett. 84, 3045 (2000).

[2] M. M. Sargsian et al., J. Phys. G 29, R1 (2003) [arXiv:nucl-th/0210025].

[3] S.J. Brodsky and B.T. Chertok, Phys. Rev. Lett. 37, 269 (1976). 
[4] K. Egiyan et al., Nucl. Phys. A 580, 365 (1994) [arXiv:nucl-th/9401002].

[5] L. L. Frankfurt et al., Z. Phys. A 352, 97 (1995) [arXiv:nucl-th/9501009].

[6] L. L. Frankfurt et al., Phys. Rev. C 56, 1124 (1997) arXiv:nucl-th/9603018.

[7] M. M. Sargsian, Int. J. Mod. Phys. E 10, 405 (2001) [arXiv:nucl-th/0110053].

[8] L. L. Frankfurt and M. I. Strikman, Phys. Rept. 160, 235 (1988).

[9] C. E. Carlson et al., Ann. Rev. Nucl. Part. Sci. 47, 395 (1997).

[10] R. A. Gilman and F. Gross, J. Phys. G 28, R37 (2002).

[11] R. Feynman, Photon Hadron Interactions, W.A. Benjamin Inc., 1972.

[12] J. Napolitano et al., Phys. Rev. Lett. 61, 2530 (1988); S.J. Freedman et al., Phys. Rev. C48, 1864 (1993).

[13] J.E. Belz et al., Phys. Rev. Lett. 74, 646 (1995).

[14] C. Bochna et al., Phys. Rev. Lett. 81, 4576 (1998).

[15] E.C. Schulte et al., Phys. Rev. Lett. 87, 102302 (2001).

[16] E. C. Schulte et al., Phys. Rev. C 66, 042201 (2002).

[17] M. Mirazita et al.[CLAS Collaboration], Phys. Rev. C 70, 014005 (2004)

[18] S.J. Brodsky and G.R. Farrar, Phys. Rev. Lett. 31, 1153; V. Matveev, R.M. Muradyan and A.N. Tavkhelidze, Lett. Nuovo Cimento 7, 719 (1973).

[19] N. Isgur and C.H. Llewellyn Smith, Phys. Rev. Lett. 52, (1984) 1080.

[20] A. Radyushkin, Acta Phys. Pol. B15, 403 (1984).

[21] M. M. Sargsian, Phys. Lett. B 587, 41 (2004) arXiv:nucl-th/0309054].

[22] M. M. Sargsian, arXiv:nucl-th/0208027.

[23] S. J. Brodsky et al., Phys. Lett. B 578, 69 (2004) arXiv:nucl-th/0305068].

[24] S.J. Brodsky and J.R. Hiller, Phys. Rev. C 28, 475 (1983).

[25] L. A. Kondratyuk et al., Phys. Rev. C 48, 2491 (1993); V. Y. Grishina, et al. Eur. Phys. J. A 10, 355 (2001).

[26] J.M. Laget, Nucl. Phys. A 497, 391 (1989).

[27] R. Gilman and E. Piasetzky (Spokespersons). JLab Experiment, E-03-101.

[28] K. Wijesooriya et al. [Jefferson Lab Hall A Collaboration.], Phys. Rev. Lett. 86, 2975 (2001).

[29] X. Jiang et al. [Jefferson Lab Hall A Coll.], Phys. Rev. Lett. 98, 182302 (2007) 